\title{
CLINICAL USES OF 2,3-DIMERCAPTOPROPANOL (BAL). V. SKIN SENSITIZATION TO BAL ${ }^{1}$
}

\author{
By MARION B. SULZBERGER, RUDOLF L. BAER, AND ABRAM KANOF \\ (From Cornell University Medical College, New York City)
}

(Received for publication February 5, 1946)

The possibility that BAL (2,3-dimercaptopropanol) (1) might be used extensively on human skins naturally raised the question of its potentialities as a skin sensitizer. Kidd in 1942 (2) reported on deliberately produced skin sensitization to BAL in guinea pigs. In 1942 we observed and described what was to our knowledge the first human case of skin sensitization to BAL (3). Somewhat later, Sparks and Levi (4) found evidence of sensitization to BAL in 13 out of 32 volunteers whose skin had been burned with liquid Lewisite, and subsequently treated with 1 or several applications of BAL solution applied to the chemical burns. Davis (5) reported dermatitis due to $\mathrm{BAL}$, or a complex containing BAL, in 11 men (18 per cent) of a series of 61 volunteers who each had one of several Lewisite burns treated with BAL solution. However, it is noteworthy that in 8 of the 11 volunteers in Davis' group, the dermatitis was not confined to the BAL-treated sites, but was present also at the other sites to which Lewisite, but not BAL, had been applied.

The evaluation of the sensitizing capacity of BAL, on the basis of the experimental work cited above, was complicated by the fact that in all the observations on human beings the application of BAL had in each instance been preceded by the local application of some other chemical agent, which had produced an inflammatory reaction in the skin. The experiments which are the subject of this report were designed to answer the following questions: Is $\mathrm{BAL}$ alone a sensitizing agent when repeatedly applied to a normal skin area of human subjects; if so, what is the incidence of such sensitization; and is the incidence of sensitization produced by BAL alone significantly different from the incidence of sensitization obtained

\footnotetext{
1 The work described in this paper was done under a contract, recommended by the Committee on Medical Research, between the Office of Scientific Research and Development and Cornell University Medical College.
}

by the application of BAL to skin areas which have been damaged previously by some other chemical agent, such as Lewisite or liquid mustard gas?

\section{EXPERIMENT I-PRODUCTION OF DELIBERATE SENSITIZATION OF HUMAN SKIN TO BAL \\ Subjects and procedure}

One hundred and two human volunteers received skin applications of BAL as follows:

Group A : 35 human subjects were given a supply of 5 per cent BAL in a grease ointment.

Group B : 35 human subjects were given a supply of 5 per cent BAL in a carbowax ointment.

Group C: 32 human subjects were given a supply of 5 per cent BAL in ethylene glycol.

All subjects were intelligent and cooperative; and all were carefully shown how to rub a small amount of the BAL preparation, which had been handed to them, into a skin area of approximately $5 \times 10 \mathrm{~cm}$. on the flexor aspect of the left forearm. No definite instructions as to the length of the rubbing or the amount to be applied were given, but the amount which was suggested and used in demonstration was about 0.3 to $0.4 \mathrm{gram}$ of BAL ointment, or 2 drops of the BAL solution delivered with a medicine dropper. The subjects themselves repeated the rubbings to the same area daily for a total of 14 applications. Interval readings were made on the 7 th day, and the final readings were made on the 15 th day. At the start of the experiment all volunteers were handed protocol forms on which they were to note the dates of the applications and the signs and symptoms, if any. The applications were to be stopped as soon as a persistent and definite erythema or other type of eruption appeared. The transitory erythema and whealing (6), which are quite regularly observed 15 minutes to 2 hours after application of BAL, were discounted.

\section{Results}

Ninety-one of the 102 subjects who started the experiment were still available for reexamination after 15 days. Three of these 91 subjects could not be included in the evaluation of the data, since the applications either had been stopped prematurely or had been carried out too infrequently and/or irregularly. 
Only persistent erythematous and papular local reactions were regarded as evidence that sensitization had taken place.

The degrees of reaction were recorded as ranging from mild to marked as follows:

Mild reactions consisted of a faint but definite erythema and/or a few isolated pinhead-sized papulo-urticarial lesions.

Marked reactions consisted of marked erythema and/or numerous confluent pinhead-sized urticarial and papular lesions.

Moderate reactions were those intermediate between the two first described.

Itching of the affected area was slight to moderate in a few of the volunteers with marked reactions; and was either negligible or absent in all others. In no case did the reactions interfere with the regular activities of the volunteers (studies, drill, physical exercise).

The results in the individual groups were:

Group $A$ ( 5 per cent BAL in grease ointment) : 31 of 35 subjects completed the experiment. Of these 31 subjects, 6 had become sensitized ( 2 markedly, 1 moderately, 3 slightly).

The dermatitis started in 1 volunteer 7 days after the first application; the dermatitis started in 1 volunteer 9 days after the first application; the dermatitis started in 1 volunteer 11 days after the first application; the dermatitis started in 3 volunteers 14 days after the first application.

Group $B$ (5 per cent BAL in carbowax ointment) : 28 of 35 volunteers completed the experiment. Of these 28 subjects, 4 had become sensitized ( 2 markedly, 1 moderately, 1 slightly).

The dermatitis started in 1 volunteer 12 days after the first application; the dermatitis started in 2 volunteers 13 days after the first application; the dermatitis started in 1 volunteer 14 days after the first application.

Group $C$ (5 per cent BAL in ethylene glycol): 29 of 32 volunteers completed the experiment. Of these 29 subjects, 6 had become sensitized ( 2 markedly, 4 slightly).

The dermatitis started in 1 volunteer 10 days after the first application; the dermatitis started in 1 volunteer 11 days after the first application; the dermatitis started in 2 volunteers 12 days after the first application; the dermatitis started in 2 volunteers 14 days after the first application.
Thus, under the conditions of this experiment, 5 per cent BAL in a grease ointment, 5 per cent $\mathrm{BAL}$ in a carbowax ointment, and 5 per cent $\mathrm{BAL}$ in ethylene glycol produced definite sensitization and sensitization dermatitis in 16 out of 88 human subjects, i.e., in 19 per cent of the subjects exposed. There appeared to be no significant difference in the sensitizing capacity of BAL when used in each of the three vehicles mentioned above.

There was no evidence that the onset of sensitization was earlier in those volunteers in whom marked reactions developed, than in those in whom the eventual reaction was slight.

Clinically the sensitization dermatitis was papulo-urticarial in appearance; definite clinical vesicles were not observed. The eruption did not spread beyond the sites of the deliberate application. In all cases the eruption subsided and disappeared without treatment within a maximum of about 5 days after the last exposure.

EXPERIMENT II-THE SPECIFICITY OF BAL SENSITIZATION ; LOCAL DIFFERENCES IN SENSITIVITY TO BAL

\section{Subjects}

Nineteen subjects participated in this experiment.

Group A: 6 subjects previously exposed and sensitized. This group consisted of 6 of the subjects who had become most strongly sensitized in Experiment I, and who were selected for further study. The further studies were begun 13 days after the last inunction of BAL-containing material, and at a time when the dermatitis had entirely disappeared in even the strongest reactors. Two of these 6 subjects had become' sensitized through application of 5 per cent BAL in grease ointment; 2 through application of 5 per cent BAL in carbowax ointment, and 2 through application of 5 per cent BAL in ethylene glycol.

Group $B: 6$ subjects previously exposed to BAL but not sensitized. This group consisted of 6 subjects who had been included in Experiment I, but who had shown no signs of dermatitis or sensitization after the 14 daily applications of BAL preperations. Two of these had been among those receiving applications of 5 per cent BAL in grease ointment, 2 had received applications of 5 per cent BAL in carbowax ointment, and 2 had received applications of 5 per cent BAL in ethylene glycol.

Group C: 7 subjects not previously exposed to BAL, and presumably not sensitized to that substance. This group consisted of 7 "fresh" subjects who had not been used in any previous experiments and who, to the best of our knowledge, had no previous exposure to BAL or its relatives. 


\section{Procedure}

Each of the 12 subjects in Groups $A$ and $B$ received an inunction of 5 per cent BAL both to the previously exposed flexor surface of the left forearm (Exp. I), and also to the not previously exposed flexor surface of the right forearm. In each individual the vehicle employed for the BAL was the same as that which had been used in Experiment $I$ in his particular case. The amount of ointment rubbed on the skin of each arm was approximately 0.3 to $0.4 \mathrm{grams}$, and the amount of liquid was 2 to 3 drops delivered with an ordinary medicine dropper. The duration of each inunction was 30 seconds.

The 7 not previously exposed control subjects (Group C) received inunctions to each forearm in the manner above described; 2 received 5 per cent BAL in grease ointment; 2 received 5 per cent BAL in carbowax ointment; and 2, 5 per cent BAL in ethylene glycol.

In addition to these inunction tests with BAL, other substances chemically related to BAL were studied in order to throw light on the specificity of the BAL reaction. The formulas of these substances are set forth in Table I. They were applied to the backs of each of the 19 volunteers in the form of both scratch and patch tests with the orthodox procedures (7). The scratch tests were read for immediate wheal reactions from 20 to 40 minutes after application, and the patch tests were read 48 hours after application.
Results

\section{Inunction tests}

The results of these tests are given in Table II. From this table it will be seen that all individuals previously recorded as sensitized in Experiment I showed unequivocal evidence of hypersensitivity of the skin of the previously exposed left forarm. This local hypersensitivity was evidenced in some instances by persistent erythema appearing within a few minutes after the inunction; and in all cases by an erythematous and edematous dermatitis at the 48 -hour reading.

In 4 subjects of this Group $A$, sensitization could be shown to have affected also the skin of the not previously exposed right arm, as evidenced by varying degrees of dermatitis on this arm also. It is noteworthy that in 3 of these 4 subjects the reaction of the previously. exposed left arm appeared earlier, and was also much more intense, than that of the not previously exposed right arm. In the 2 remaining subjects in Group A, the right arm showed no reaction to the inunction, although there was a reaction on the previously exposed left arm.

TABLE I

$B A L$ and other compounds (dithiols) tested in Experiment II<smiles>CC(S)CO</smiles>

BAL<smiles>CC(=O)OCC(S)CS</smiles>

Compound 4<smiles>CC(CO)CS</smiles>

Compound 2<smiles>OCCCCCCCCCNCCSCC(S)CO</smiles>

Compound 5<smiles>NC(=O)NCC(S)CS</smiles>

Compound 7<smiles>SCC(S)CS</smiles>

Compound 3 .<smiles>CCCCCCCCCS</smiles>

Compound 6 


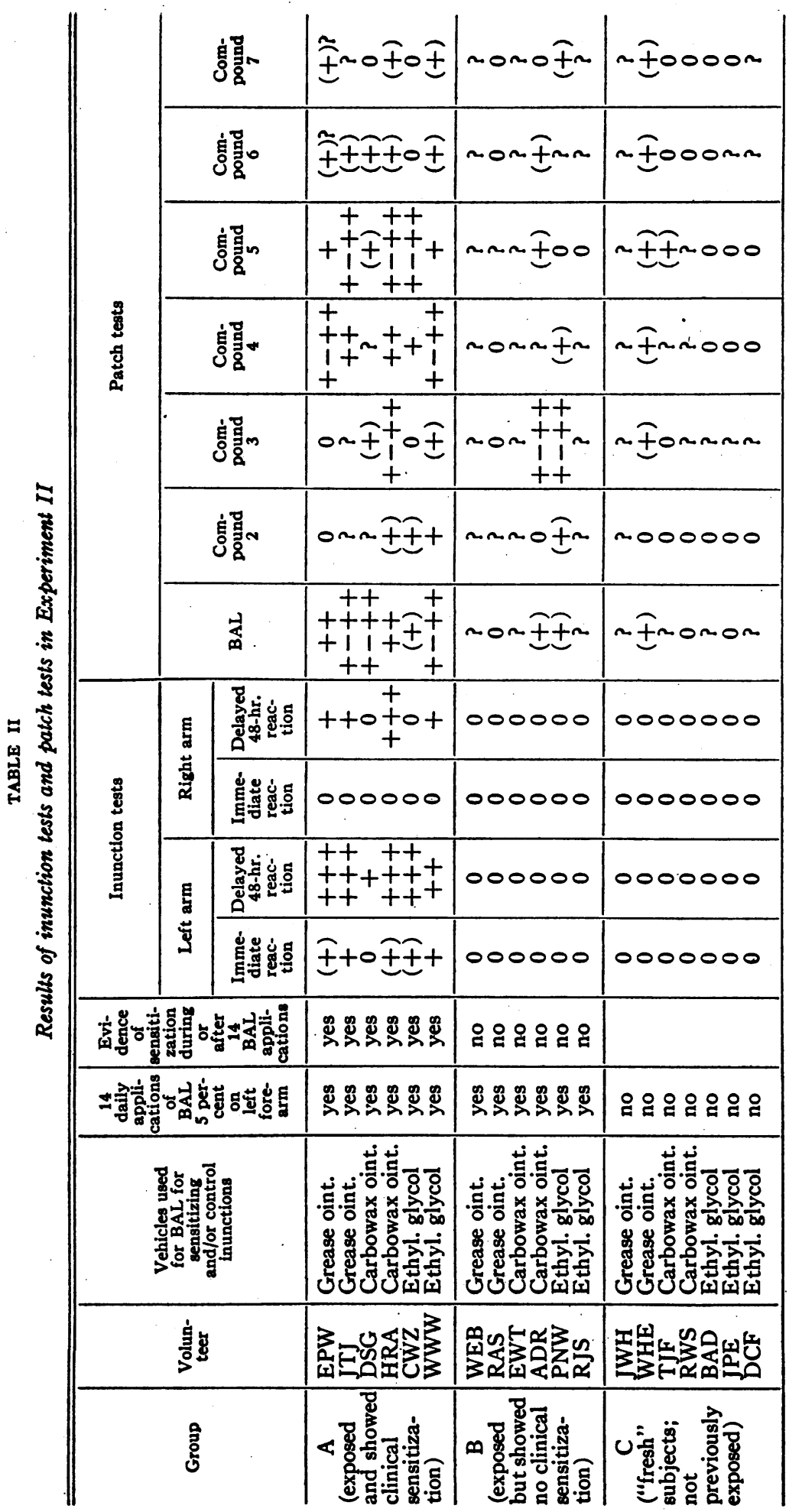


The dermatitis produced by the inunctions in Group A was erythematous and edematous with some mottling and papule formation. Vesicular lesions, characteristic of the eczematous contacttype of allergic sensitization, were not observed.

In the subjects of Group B and Group C, there were no reactions to inunctions of $\mathrm{BAL}$ materials on either arm, i.e., no evidences of sensitization.

These results demonstrate that the 6 subjects in Group A still showed evidence of sensitization to BAL. In each of the 6 subjects, there were marked differences in the degree of sensitization produced at different skin sites. The previously exposed skin sites on the left arm were on the whole significantly more sensitive than the not previously exposed skin sites on the right arm; in 2 of the subjects, sensitization of the not previously exposed right arm was either absent, or so slight that it did not become clinically manifest under the conditions of these inunction tests. The significance of these results is indicated by the complete absence of reactions in Groups B and C.

\section{Scratch tests}

The scratch tests performed by the orthodox techniques produced variable degrees of erythema in different subjects. None of the compounds elicited greater response in Group $A$ than in Group B and Group C. Moreover, there was no greater response to the scratch tests with BAL than to any of the other substances tested. Thus there was no evidence of sensitization of urticarial type demonstrable by this form of testing.

\section{Patch tests}

The results of the patch tests performed with orthodox techniques are included in Table II. Five of the 6 subjects in Group A gave definite reactions to BAL. The 1 subject in Group $A$ who failed to give a definite reaction to BAL was 1 of the 2 subjects who had failed to react to the inunction test on the not previously exposed right arm. Obviously, in this subject the sensitization to BAL was even more localized than in the other 5 subjects of Group A.

In addition to the positive patch tests with BAL, 5 subjects in Group A gave definite reactions to compound 4 , and 5 gave definite reactions to com- pound 5. There were no significant reactions to either of these compounds in the subjects of Groups B and C. The conclusion therefore seems warranted that there were cross-reactions with compounds 4 and 5 in the subjects sensitized to BAL.

There was 1 significant reaction to compound 3 in Group A, and 2 significant reactions in Group $B$; and there was no reaction in Group C. This finding suggests the possibility, that there are cross-reactions to compound 3 in subjects sensitized to BAL. The single positive reaction to compound 1 is likewise insufficient to be regarded as evidence of cross-reaction. The patch test reactions clinically closely resembled the morphe of the reactions to inunction tests. They were all erythematous; or erythematous, papular and edematous; but never vesicular.

\section{EXPERIMENT III-INCIDENCE OF SENSITIZATION TO} BAL AFTER ITS APPLICATION ON DAMAGED SKIN

\section{Subjects}

Sixty-six subjects participated in this experiment.

Group $A$ : 53 subjects who had been burned with mustard gas and who were known to have had previous exposures to BAL. The interval between first exposure to BAL and the time of application of the tests for sensitization varied from 9 to 33 days. In the 53 subjects of this group, the preceding applications of BAL were as follows :

In each subject an area on the flexor aspect of each forearm about $3.0 \times 5.0 \mathrm{~cm}$. to $6.0 \times 8.0 \mathrm{~cm}$. had been damaged by burning with liquid mustard gas. The center of the damaged area had sustained what was largely a third degree burn, but the peripheral areas were the site of either second and/or first degree burns.

Either 5 per cent BAL in grease ointment, 5 per cent BAL in carbowax ointment, 10 per cent BAL in petrolatum, or undiluted BAL had been applied 1 or more times to the damaged skin sites. In some of the subjects BAL had been applied to the damaged area on only one arm; in the remaining subjects, BAL had been applied to the damaged area on both arms.

Undiluted BAL was used in the form of a wet dressing left on for 48 hours. The BAL ointments were used as ointment dressings left on for periods varying in different volunteers from 48 hours to 7 days; or they were massaged into the damaged site 1 to 6 times at hourly intervals; or they were bandaged on following 1 application of a few drops of undiluted BAL.

Group $B: 13$ subjects who had no known previous exposure to BAL. 


\section{Procedure}

Each subject received patch tests with 5 per cent BAL in grease ointment, and with this ointment vehicle alone (without BAL). The patch tests were applied to the grossly normal skin on the upper back. Furthermore, each subject received inunction tests with these two ointments. One-twentieth $\mathrm{ml}$. of each ointment was applied to an area on the back, and then rubbed in for 30 seconds with a glass rod.

In those volunteers who had received applications of liquid BAL and/or BAL ointment only to the damaged site on one arm, the patch and inunction tests were carried out on the same side of the back, i.e. if a subject had had BAL applied to the left arm site only, both patch and inunction tests were done on the left side of the back.

\section{Results}

The results of this experiment are summarized in Table III. From this table it will be seen that among the 53 subjects in Group A, 35 (66 per cent) gave positive reactions to patch tests, and 27 (51 per cent) to inunction tests with BAL ointment. Among the 14 subjects who had been exposed previously on only one arm, 5 (36 per cent) gave positive reactions to patch tests; and 4 (29 per cent), to inunction tests with BAL ointment. Among the 39 subjects who had been exposed previously to BAL on both arms, 30 ( 77 per cent) gave positive reactions to patch tests; and 23 ( 59 per cent), to inunction tests with BAL ointment.

In none of the 13 subjects in the not previously exposed Group B were positive reactions elicited with BAL ointment in either patch or inunction tests. In none of the subjects of Groups $A$ and $B$ were positive reactions elicited by the ointment base alone in either patch or inunction tests.
EXPERIMENT IV-INCIDENCE OF SKIN SENSITIZATION TO BAL AFTER ITS PARENTERAL ADMINISTRATION

\section{Subjects}

Eighteen subjects participated in this experiment. These subjects had received 4 to 8 intramuscular injections of a solution of 10 per cent BAL and 20 per cent benzyl benzoate in peanut oil (6). The last injection of BAL had been given 13 to 25 days prior to the performance of the skin tests. The total dosage of BAL which had been administered intramuscularly varied from $930 \mathrm{mgm}$. to $2300 \mathrm{mgm}$. None of the subjects had had known skin exposure to $\mathrm{BAL}$, except for the possible leaking of $\mathrm{BAL}$ solution onto the skin through the needle tract after the intramuscular injections.

\section{Procedure}

Each subject received patch tests with 5 per cent BAL in grease ointment, and 5 per cent BAL in carbowax ointment. The patch tests were applied to the grossly normal skin on the upper back. No control group of not previously exposed subjects was used in this experiment, since it had been shown previously, in other experiments, that the two BAL ointments employed produced no significant reaction when applied as patch tests to normal subjects not previously exposed to BAL.

Four of these subjects received a further intramuscular injection of $450 \mathrm{mgm}$. of BAL after the patch test reactions were read.

\section{Results}

Five of the 18 subjects showed evidence of skin sensitization to BAL in the form of positive reactions to the patch tests. Nine of the 18 subjects showed no reaction, while 4 showed reactions which were not sufficiently pronounced to be regarded as evidence of sensitization.

TABLE III

Incidence of sensitization to BAL after its application to damaged skin (Experiment III)

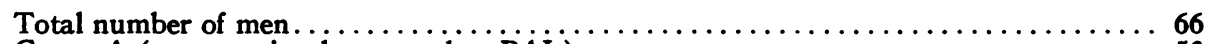

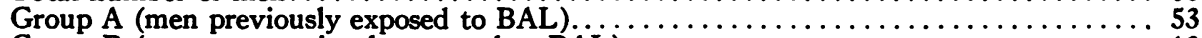

Group B (men not previously exposed to BAL) $\ldots \ldots \ldots \ldots \ldots \ldots \ldots \ldots \ldots \ldots \ldots \ldots \ldots$

\begin{tabular}{l|c|c|c|c}
\hline \hline & \multicolumn{2}{|c|}{ Inunction test positive } & \multicolumn{2}{c}{ Patch test positive } \\
\cline { 2 - 5 } & Blank ointment base & BAL ointment & Blank ointment base & BAL ointment \\
\hline 53 men in Group A (previously exposed) & $0=0$ per cent & $27=51$ per cent & $0=0$ per cent & $35=66$ per cent \\
$\begin{array}{l}39 \text { men in Group A (previously exposed on } \\
\text { both arms) }\end{array}$ & $0=0$ per cent & $23=59$ per cent & $0=0$ per cent & $30=77$ per cent \\
$\begin{array}{l}14 \text { men in Group A (previously exposed on } \\
\text { one arm) }\end{array}$ & $0=0$ per cent & $4=29$ per cent & $0=0$ per cent & $5=36$ per cen $t$ \\
13 men not previously exposed & $0=0$ per cent & $0=0$ per cent & $0=0$ per cent & $0=0$ per cent \\
\hline
\end{tabular}


The clinical appearance, degree and intensity of the skin sensitization which resulted from the intramuscular injections of BAL, appeared to be similar to that observed in individuals who had become sensitized after repeated external applications of BAL to normal skin. It was noteworthy that the renewed intramuscular injection of $450 \mathrm{mgm}$. of BAL produced no cutaneous reaction or dermatitis in 4 of these subjects whose skin had become sensitized following the course of intramuscular injections of BAL given 15 to 27 days previously.

\section{COMMENT}

The results of these experiments clearly demonstrate some of the factors which determine the incidence of sensitization, as well as the level of sensitivity. We found an incidence of 19 per cent sensitization to BAL in individuals who applied BAL ointment or BAL solution to a site on one forearm daily for a total of 14 daily applications. This might be called the sensitizing capacity or sensitizing index of BAL on normal skin $(8,7)$. When BAL is applied repeatedly or for a prolonged period of time to a damaged area on one arm the sensitizing capacity of BAL is much higher, namely 36 per cent. After repeated or prolonged application to burned areas on both arms, the sensitizing capacity rose to 77 per cent. These results suggest that the incidence of sensitization is influenced not only by the number of applications and the concentration of the allergen used, but also by the size of the skin area exposed to the allergen, and perhaps also by the total quantity of allergen applied.

The finding that a contact-type sensitizing agent will produce a higher incidence of sensitization on inflamed or burned skin has previously been reported $(9,10)$. However, these tests with $\mathrm{BAL}$ are the first systematic experimental demonstration in human subjects of the difference in sensitizing capacity of an allergen when applied to grossly normal and to deliberately damaged skin. The most acceptable hypothesis for this increase in incidence of sensitization is that the presence of devitalized tissue at the site of application permits the ready combination of the smaller BAL molecules with proteins or other larger molecules, and thus accelerates the formation of complexes with a high capacity to sensitize. (For purposes of comparison, the figures on incidence of sensitization are here given as percentages, despite the fact that the size of the series used may not be entirely satisfactory from a statistical viewpoint.)

These experiments demonstrated another interesting factor which has, to our knowledge, not previously been systematically studied in series of deliberately sensitized human subjects. We refer to the local differences in the degree of sensitivity at, or adjacent to, the site of sensitizing exposure, and at more distant skin areas. That there are very great local differences in the degree of sensitivity to contact-type allergens has been known for many years, since J. Jadassohn first called attention to the occurrence of such a phenomenon (11). It has more recently been discussed by Stauffer (12) and Sulzberger and Kerr (13).

Various degrees of spread (or lack of spread) of sensitization to BAL were observed. Thus in Experiment II all 6 subjects in Group A showed unequivocal evidence of sensitization on the previously exposed left forearm. In 2 of these 6 subjects, no evidence of sensitization could be demonstrated on the not previously exposed right forearm. In one of these 2 subjects, the sensitization appeared to be so localized that even a patch test with BAL on the upper back was negative. In 3 of the 6 subjects the not previously exposed forearm had become sensitized, but to a significantly weaker degree than the previously exposed forearm; while in 1 subject both forearms were equally sensitized.

Experiment IV showed that the skin can become hypersensitive to BAL subsequent to parenteral injection of this agent. It was further found that 4 subjects who had become skin hypersensitive to BAL (as demonstrated by positive patch tests), after repeated intramuscular administration, showed no cutaneous effects whatsoever when a large dose of BAL was again administered intramuscularly. Whether this phenomenon, which is obviously of considerable practical and theoretical significance, occurs in all cases of cutaneous sensitization to BAL cannot be decided on the basis of the 4 cases studied by. us.

The type of skin sensitization produced by BAL as seen in our experiments is neither the classic eczematous contact-type (characteristic example: poison ivy sensitization) nor the classic urticarial type (characteristic example: sensitization to 
"protein" fraction of ragweed pollen). In contrast to the usual forms of experimental contacttype eczematous sensitization, no vesicular reactions were seen either clinically nor in patch or inunction tests. Furthermore, there was a marked tendency for the BAL sensitivity to be significantly greater in the sites of actual previous exposure; and perhaps as a corollary to this, the dermatitis produced by BAL did not show the tendency to "spontaneous" spread or dissemination which is so commonly seen in many forms of eczematous sensitizations.

However, the BAL sensitization resembled contact-type eczematous sensitization in that the reactions were produced by external contact with the allergen, and the patch test reactions were positive after 24 to 48 hours, while the scratch tests for immediate wheal reactions were negative. In these last 2 respects, the BAL sensitization differs from the classic urticarial type of skin sensitization as seen, for example, in hayfever, asthma and atopic dermatitis ; for in these the immediate wheal reaction is generally positive, and the response to the patch test is generally negative. The histologic examination of these reactions might have given further information on the type of sensitization produced. Unfortunately it was not possible to obtain biopsies from the reaction sites in our subjects.

The evidence at hand suggests that the sensitization produced to BAL is neither the classic contact-type of vesicular dermatitis, nor the classic urticarial form, but a somewhat different form of allergic response consisting of erythema, edema and papules upon external exposure of the skin to the allergen.

The cross reactions which BAL-sensitized subjects gave when tested with compounds 4 and 5 are interesting in that these 2 compounds are dithiol derivatives from which one would expect the original dithiol, i.e., BAL, to be liberated rather readily in the tissues.

\section{SUMMARY AND CONCLUSIONS}

1. When applied to normal undamaged skin, 5 per cent BAL in grease ointment, 5 per cent $\mathrm{BAL}$ in carbowax ointment, and 5 per cent BAL in ethylene glycol all produced definite sensitization and sensitization dermatitis in 16 out of 88 human subjects, i.e., in 19 per cent of the subjects exposed. There appeared to be no significant difference in the sensitizing capacity of BAL when used in these 3 vehicles.

2. When applied to damaged (chemically burned) skin, 5 per cent and 10 per cent BAL ointments and/or undiluted BAL produced definite sensitization and sensitization dermatitis in 35 out of 53 human subjects, i.e., in 66 per cent of the subjects exposed.

3. Although the series are too small to permit definite conclusions, our results suggest that there may be a significant difference in incidence of sensitization between those subjects who received BAL applications to the damaged (burned) site on only one arm (5 of 14 subjects, i.e., 36 per cent sensitized) and those who received BAL applications to burned sites on both arms ( 30 of 39 subjects, i.e., 77 per cent sensitized).

4. The above results may be explained by the fact that the presence of devitalized tissue at the site of application in burned area permits the ready combination of BAL with proteins, or other larger molecules, and the formation of complexes with a high capacity to sensitize.

5. The sensitivity produced by BAL was neither the classic eczematous contact-type nor the classic urticarial type of skin sensitivity. It differed somewhat from both of these common types and consisted of erythema, edema and papules upon external exposure of the skin to the allergen.

6. While the sensitivity to BAL did not remain strictly confined to the areas of exposure, there was evidence that in the majority of individuals the exposed area was more strongly sensitive than other parts of the skin. There was evidence that in exceptional cases the sensitivity may remain practically restricted to the previously exposed parts.

7. The sensitivity produced by BAL was not confined strictly to BAL, for the majority of sensitized individuals reacted also to patch tests with at least 2 chemically related compounds.

8. The repeated intramuscular injection of BAL produced skin sensitization as demonstrated by positive patch tests in 5 of 18 subjects. When a large dose of BAL was again injected intramuscularly, in 4 of these subjects whose skin had become hypersensitive to BAL, no cutaneous reactions were produced. 


\section{BIBLIOGRAPHY}

1. Waters, L. L., and Stock, C. C., BAL (British AntiLewisite). Science, 1945, 102, 601.

2. Kidd, J., 1942, Personal communication.

3. Barr, D. P., and Sulzberger, M. B., 1942, Unpublished.

4. Sparks, A. L., and Levi, J. E., 1943, Personal communication.

5. Davis, M. I. J., 1943, Personal communication.

6. Sulzberger, M. B., Baer, R. L., and Kanof, A., Studies on the toxicity of BAL on percutaneous and parenteral administration. J. Clin. Invest., 1946, 25, 474.

7. Sulzberger, M. B., and Baer, R. L., Year Book of Dermatology, 1944, p. 13. Year Book Publishers, Chicago, IIl.

8. Sulzberger, M. B., and Simon, F. A., Arsphenamine hypersensitiveness in guinea pigs. III. Experiments demonstrating (a) the regional geographic variability in susceptibility to sensitization; (b) the chemical specificity of the hypersensitivity; and (c) the variation in the sensitizing proclivities (sensitization index) of different brands. J. Allergy, 1934, 6, 39.

9. Sulzberger, M. B., and Wise, F., Drug eruptions. II. Dermatitis eczematosa due to drugs. Arch. Dermat. and Syph., 1933, 28, 461.

10. Landsteiner, K., and Di Somma, A. A., Studies on the sensitization of animals with simple chemical compounds. VIII. Sensitization to picric acid: subsidiary agents and mode of sensitization. $\mathrm{J}$. Exper. Med., 1940, 72, 361.

11. Jadassohn, J., Zur Kenntnis der Medikamentoesen Dermatosen. Verh. d. V. Deutsch. Dermat. Gesellsch., Graz. 1895.

12. Stauffer, H., Die Ekzemproben (Methodik u. Ergebnisse). Arch. f. Dermat. u. Syph., 1931, 162, 517.

13. Sulzberger, M. B., and Kerr, P., Sensitizations of eczematous type: ten selected cases illustrating some uses of the patch test. J. Allergy, 1933, 4, 326. 